\title{
Tumor suppressive microRNA-138 inhibits metastatic potential via the targeting of focal adhesion kinase in Ewing's sarcoma cells
}

\author{
KAZUHIRO TANAKA, MASANORI KAWANO, ICHIRO ITONAGA, TATSUYA IWASAKI, \\ MASASHI MIYAZAKI, SHINICHI IKEDA and HIROSHI TSUMURA \\ Department of Orthopaedic Surgery, Faculty of Medicine, Oita University, Oita 879-5593, Japan
}

Received October 27, 2015; Accepted December 14, 2015

DOI: $10.3892 /$ ijo.2016.3317

\begin{abstract}
Short non-coding RNAs, called microRNAs (miRNAs), regulate cell biology by affecting the expression of target genes. However, we know little about the miRNAs regulating the growth and progression of Ewing's sarcoma (ES). To identify possible oncogenic factors in ES, we used a microarray-based approach to profile the changes in the expression of miRNAs and the downstream mRNAs in five ES cell lines. One miRNA, miR-138, was significantly downregulated, whereas the expression of focal adhesion kinase (FAK) was significantly upregulated in all tested ES cells. When miR-138 was transfected into ES cell lines, the expression of FAK in these cells was greatly suppressed and inhibited the proliferation and mobility of ES cells. Overexpression of miR-138 in vitro resulted in further inhibition of the cell cycle at the G1 phase and in the induction of anoikis, in a dose- and time-dependent manner. Moreover, miR-138 overexpression in ES cells significantly suppressed the number of distant metastases in vivo. The data in the present study demonstrates for the first time a novel mechanism that regulates the expression of FAK via miR-138 in ES cells.
\end{abstract}

\section{Introduction}

Ewing's sarcoma (ES) is the second most common bone malignancy after osteosarcoma (1). ES is characterized by a rapid proliferation of small round tumor cells, which causes rapid and extensive progressive bone destruction in children, adolescents and young adults (2). With advances in adjuvant therapy, the survival rate of patients with a localized form of the disease has reached $\sim 70 \%$ (3). However, patients with a metastatic, recurrent form of the disease have a poor prognosis (4).

Correspondence to: Dr M. Kawano, Department of Orthopaedic Surgery, Faculty of Medicine, Oita University, Oita 879-5593, Japan E-mail: kawano@oita-u.ac.jp

Key words: miR-138, focal adhesion kinase, Ewing's sarcoma
MicroRNAs (miRs) are evolutionarily conserved, small, non-coding RNA molecules of 22 nucleotides in length which can play critical roles in cell proliferation, apoptosis, and developmental timing by negatively regulating the stability or translational efficiency of their target mRNAs (5). Global profiling and functional studies have demonstrated that multiple miRNAs contribute to cancer development and biology $(6,7)$. MicroRNA-138 is downregulated in different cancers, including prostate, ovarian, and lung cancer $(8,9)$. A recent study has identified that miR-138 suppresses cancer cell proliferation or motility by targeting BCR-ABL/GATA-1 in chronic myeloid leukemia (10), cyclin D1 in nasopharyngeal carcinoma (11) and SOX4 and HIF-1 $\alpha$ in ovarian cancer (12). It has been reported that the expression level of miR-138 in several types of cancers is reduced compared to normal tissues, and that miR-138 can prevent the cellular proliferation or motility via the downregulation of oncogenes. However, the role of miR-138 in the proliferation and invasiveness of ES remains unclear.

Focal adhesion kinase (FAK) is an important receptor at the proximal position in the regulation of growth factor- and integrin-stimulated cell motility. Specifically, it plays a key role in focal contact formation as well as turnover, and FAK may also function in promoting cell invasion and may regulate cell adhesion, migration, survival, proliferation, and differentiation in a variety of cells $(13,14)$. Some previous studies have strongly implicated FAK in the development and progression of breast and other cancers $(15,16)$.

Although several miRNAs have been found to target FAK, including miR-7 (17), miR-488 (18), and miR-151 (19), the correlation of FAK expression and miRNAs in ES cells is totally unknown.

In the present study, we analyzed the genome-wide expression of both miRNAs and mRNAs in five human ES cell lines and human mesenchymal stem cells (hMSCs). The results suggest that the expression of miR-138 was repressed, whereas that of FAK was increased, in all five ES cell lines. Based on the inverse correlation between miR-138 and FAK expression, we hypothesized that the effect of FAK in ES cells might be mediated, at least in part, by miR-138. The purpose of our study was to assess whether the expression of FAK is regulated by miR-138, and whether the pathway plays a role in tumorigenesis in ES cells. 


\section{Materials and methods}

Ethics statement. All animal experiments were performed under the guidelines for animal experiments as stipulated by the Oita University Graduate School of Medical Science. All animal procedures carried out in this study were reviewed, approved, and supervised by the Oita University Graduate School of Medical Science, Japan. All animals were kept and the experiments were performed in accordance with the European Community guidelines for the use of experimental animals (86/609/EEC).

Cell lines. The human ES cell lines, SKES1, RDES, SKNMC and SCCH were obtained from JCRB Cell Bank (Tokyo, Japan), and WE68 was kindly provided by Dr Frans van Valen (Westfalische-Wilhelms University, Münster, Germany). Human mesenchymal stem cells (hMSCs) were purchased from Takara Biotechnology (Otsu, Japan). RDES and SKNMC cells were cultured in Dulbecco's modified Eagle's medium (DMEM) high glucose medium (Invitrogen, NY, USA) with $10 \%$ FBS and $1 \%$ penicillin and streptomycin. SKES1 cell were cultured in RPMI-1640 (Invitrogen) supplemented with 10\% FBS. SCCH cells were grown in minimal essential medium (MEM) supplemented with 10\% fetal bovine serum (FBS; Invitrogen) and $0.1 \mathrm{mmol} / 1$ nonessential amino acids (NEAA). hMSCs were cultured mesenchymal stem cell basal medium, chemically defined (MSCBM-CD) with MSCGM-CD SingleQuats (Takara Bio). The cells were maintained at $37^{\circ} \mathrm{C}$ incubator supplied with $5 \% \mathrm{CO}_{2}$ and passaged every 2-3 days.

RNA isolation. mRNAs were prepared from the triplicate cell cultures using RNeasy (Qiagen, Valencia, CA, USA) kit according to the manufacturer's instructions. The RNA quality was ensured, before labeling, using RNA 6000 Nano kit and Bioanalyzer 2100 (Agilent, Santa Clara, CA, USA). miRNAs were prepared from triplicate cell cultures using the miRNeasy Mini kit (Qiagen) according to the manufacturer's instructions.

Genome-wide miRNA expression microarray. GeneChip miRNA 3.0 array (Affymetrix, Santa Clara, CA) was used for miRNA expression profiling in five ES cell lines and hMSCs. The data were extracted from the images, quantile-normalized, summarized (median polish), and log2-transformed with miRNA QC software (Affymetrix). GeneSpring GX 11.0 (Agilent) was used to analyze the array results. The gene list was filtered with a fold-change cutoff of 2 resulting in the output of a list of genes that have significant differential expression at 2-fold or more. Pathway analysis was performed using KEGG pathway database (http://www.genome.jp/kegg/ pathway.html).

Analysis of mRNA expression by cDNA arrays. GeneChip Genome HG U133 Plus 2.0 Array (Affymetrix) was used for mRNA expression profiling in 5 ES cell lines and hMSC. Biotinylated cRNA was synthesized from total RNA using the 3' IVT Express kit (Affymetrix) according to the manufacturer's protocols. The entire experiment was performed twice. The microarray numerical values were analyzed using the GeneSpring GX 11.0 software, according to the RAM16 Algorithm: quantile normalization, filter by flags (detected), filter by expression on the normalized data (20.0-100.0th percentile). The gene list was filtered with a fold-change cutoff of 2, resulting in output of list with genes that have significant differential expression at 2-fold or more.

Prediction of binding site and mature miRNA transfection. Among the predicted target genes of miR-138 in the TargetScan (http://www.targetscan.org/), DIANA (http://diana.cslab.ece. ntua.gr/microT/), and PicTar (http://pictar.mdc-berlin.de/) databases, we found that FAK was one of the top candidates. One day prior to the transfection, cells were seeded onto 6-well plates $\left(5 \times 10^{4}\right.$ cells/well) and incubated with the complete medium without antibiotics $(2 \mathrm{ml} /$ well $)$. The transfection of miR-138-1-3p mimic (5'-GCUACUUCACAACACCAGG GCC-3'), miR-138-1-3p mutant (5'-CAGAUGAGCACAACAC CAGGGCC-3') and negative control (NC) mRNAs (Invitrogen) was performed using Lipofectamine 2000 reagent (Invitrogen) in antibiotics-free OptiMEM (Invitrogen) according to the manufacturer's instructions. After 48-h incubation following the transfection, the cells were harvested and processed for further analysis.

RNA extraction, $c D N A$ synthesis, and quantitative real-time $P C R$. Total RNA was extracted from prepared treated samples with the TRizol reagent (Invitrogen) and cDNA was synthesized according to the manufacturer's protocol (Roche). Quantitative real-time PCR (qRT-PCR) was performed using a Light Cycler 480 Probe Master system (Roche), and PCR-specific amplification was conducted in the LightCycler ${ }^{\circledR}$ Nano (Roche). The relative expression of genes (FAK and $\beta$-actin) was calculated with the $2^{-\Delta \Delta \mathrm{Ct}}$ method. The primers used are listed here (qRT-PCR; FAK-forward 5'-CCCAGAA AGAAGGTGAACG-3', FAK-reverse 5'-GGTCGAGGGCAT GGTGTA-3'; GAPDH-forward 5'-CCTCTATGCCAACACA GTGC-3', GAPDH-reverse 5'-GTACTCCTGCTTGCTGA TCC-3'.

Knockdown of FAK expression using siRNA. siRNA oligonucleotides targeting FAK mRNA was purchased from Ambion (Tokyo, Japan) and MISSION siRNA Universal Negative Control was purchased from Sigma-Aldrich (Osaka, Japan). The siRNAs were transfected into SKES1 and RDES cells using Lipofectamine 2000 reagent according to the manufacturer's instructions. The cell lines were harvested $48 \mathrm{~h}$ after the transfection, then subjected to various analyses. The experiment was repeated three times.

Cell proliferation assay. The cells were plated in 6-well plates $\left(5 \times 10^{4}\right.$ cells per well), and were transfected with or without hsamiR-138-1-3p mimic, negative control miRNA, FAK-siRNA or negative control-siRNA. Then the cells were incubated in antibiotic-free OptiMEM. After $48 \mathrm{~h}$ of the cultivation, the cells were counted using TC10 Automated Cell Counter (Bio-Rad).

Western blot analysis. Whole cell lysates were prepared from the cancer cell lines and cellular protein $(15 \mu \mathrm{g})$ was resolved on a precast $10 \%$ Tris- $\mathrm{HCl}$ Criterion 10-well gel (Bio-Rad) at $200 \mathrm{~V}$ (300 mAmp) for $30 \mathrm{~min}$. Antibodies rabbit anti-human FAK (\#3285), RhoA (\#2117), paxillin (\#2542), phosphoryla- 
tion (p)-paxillin (\#2541), Src (\#2108), p-Src (\#5473), Rac (\#4651), p-Rac (\#2461), PARP (\#9542), cleaved PARP (\#5625) were obtained from Cell Signaling Technology. Rabbit antiF-actin (ab130935), anti-p-RhoA (ab41435) and anti-human $\beta$-actin (ab16039) Abcam Technology (Cambridge, UK). Immunocomplexes were visualized with horseradish peroxidase-conjugated anti-rabbit immunoglobulin $\mathrm{G}$ antibodies (GE Healthcare, Tokyo, Japan), the blots were developed using ECL Plus system (GE Healthcare) with a ChemiDoc camera (ImageQuant LAS 4000 mini; GE Healthcare). The quantification of western blot signals was performed by the densitometry with ImageQuant TL software (GE Healthcare). All primary antibodies were used at a 1:1,000 dilution. Peroxidase-conjugated anti-Rabbit IgG secondary antibodies (GE Healthcare) were used at a 1:5,000 dilution. Three independent experiments were performed for each analysis and the gels have been run under the same experimental conditions.

Cell motility and migration assays. For the motility assays, stable monoclonal cell lines (SKES1/miR-negative control, SKES1/miR-138 and SKES1/siRNA-negative control, SKES1/ FAK-siRNA) and $2 \times 10^{5}$ cells transfected from stable cell lines were resuspended in the migration medium (medium without FBS), and were plated in the top chamber without the Matrigel-coated membrane (24-well insert; $8 \mathrm{~mm}$ pore size; BD Biosciences). The lower compartment was filled with $600 \mathrm{ml}$ of a medium containing $30 \% \mathrm{FBS}$ as a chemoattractant. Following an incubation for $16 \mathrm{~h}$ at $37^{\circ} \mathrm{C}$ and $5 \% \mathrm{CO}_{2}$ in a humidified incubator, the cells on the lower surface of the filter were fixed in $4 \%$ formaldehyde for $20 \mathrm{~min}$, then stained with Giemsa stain for $10 \mathrm{~min}$ at room temperature and examined by light microscopy.

Cell cycle analysis. SKES1 cells were seeded at a density of $5 \times 10^{5}$ cells/well in a 6 -well plate and transfected with miR-138 and FAK-siRNA. After transfection for $48 \mathrm{~h}$, samples were trypsinized, resuspended in PBS (Life Technologies), and fixed with cold $70 \%$ ethanol overnight. For cell cycle analysis, cells were stained with propidium iodide using Cycletest Plus DNA Reagent kit (BD Biosciences) following the manufacturer's protocol, and the cell cycle distribution was analyzed by FACS Verse flow cytometer (BD Biosciences). The percentages of cells in G0/G1, S, and G2/M phases were counted and compared. The experiments were carried out in triplicate.

Apoptosis assay. The changes in the expression of apoptotic proteins were analyzed by western blot analysis using antibodies against PAR/poly(ADP-ribose) polymerase (PARP) (\#9542) and cleaved PARP (\#9541) (Cell Signaling Technology, Tokyo, Japan) as an index of apoptosis. The FITC Annexin V Apoptosis Detection kit (BD Pharmingen, San Jose, CA, USA) was used to detect apoptotic cells by flow cytometry. Annexin $\mathrm{V}$ binding buffer was used to resuspend cells, and the cell suspensions were stained with FITC-Annexin V and PI staining solution for $15 \mathrm{~min}$ at room temperature. The samples were analyzed by FACSVerse using FACSuite analysis software (BD Bioscience). As a positive control for apoptosis, SKES1 cells treated with doxorubicin at $40 \mathrm{mg} / \mathrm{ml}$ for $20 \mathrm{~h}$ were used.
In vivo experimental and spontaneous metastasis models. The experimental metastasis model was established by injection of SKES1 cells $\left(1 \times 10^{6}\right)$ transfected with miR-138 suspended in $100 \mu \mathrm{l}$ of normal saline into the tail veins of nude mice. Four groups were established: i) untreated $(n=7)$; ii) transfection of negative control-miRs $(n=7)$; iii) transfection of miR-138 $(n=7)$ and iv) transfection of FAK-siRNA $(n=7)$. All mice were fed in standard condition with weight monitoring and sacrificed 6 weeks after the cell inoculation. Tumor volumes were measured using a micro-CT apparatus (R_mCT) which allows us to obtain high-resolution CT images in small living animals. The tumor volume of the lung nodule was estimated using the formula ( $\pi \mathrm{x}$ long axis $\mathrm{x}$ short axis $\mathrm{x}$ short axis)/6.

Statistical analysis. Statistical analysis was carried out and all analyses were conducted using SPSS ${ }^{\circledR} 18.0$ software (SPSS Japan Inc., Tokyo, Japan). Two-tailed Student's t-test was used for analysis of continuous variables. $\mathrm{P}<0.01$ was considered to be statistically significant. We determined the difference in $>3$ groups using a nonrepeated measures analysis of variance (ANOVA) and Scheffe test. Results were expressed as the mean \pm standard deviation, and $\mathrm{P}<0.01$ was considered as statistically significant.

\section{Results}

Downregulation of miR-138 expression in ES cell lines. Genome-wide miRNA expression profiling was carried out using five ES cell lines, to identify miRNAs expressed in ES cells specifically. The array analysis showed that the expression of 1,054 miRNAs in ES cells was changed (foldchange $>2.0$ ) compared to the hMSCs (Fig. 1A). Among these 1,054 miRNAs, 228 were significantly upregulated, whereas 705 were significantly downregulated, in all tested ES cells compared to the hMSCs. The remaining 121 miRNAs were up- or downregulated among the five ES cell lines. In the ES cell lines, a decrease in the expression of miR-138 by 4.03 179.82-fold was observed compared to the hMSCs. The rank of fold-change of miR-138 were 66 out of 705 for SCCH, 97 out of 705 for WE68, 217 out of 705 for SKES1, 399 out of 705 for RDES and 531 out of 705 for SKNMC.

Upregulation of FAK expression in ES cell lines. The cDNA array analysis demonstrated that the expression of 3,043 mRNAs was significantly changed between five ES cell lines and hMSCs (Fig. 1B). We found that 1,062 genes were significantly upregulated, whereas 1,884 genes were significantly downregulated, and the remaining 97 genes were up- or downregulated in five ES cell lines compared to hMSCs. The expression of FAK was increased by $2.45-5.07$-fold in the five ES cell lines.

miR-138 directly targets FAK $m R N A$. The region complementary to the miR-138 seed region was found in the 3'-UTR of human FAK (Fig. 2A). To test whether miR-138 expression affected endogenous FAK expression, we transfected the miR-138-1-3p and miR-138-1-3p mutant oligonucleotides, (Fig. 2B) as well as the negative control-miR, into SKES1 cells. We observed an increased miR-138 expression by 5.32 -fold compared with control-miR (Fig. 2C) and decreased FAK 
A

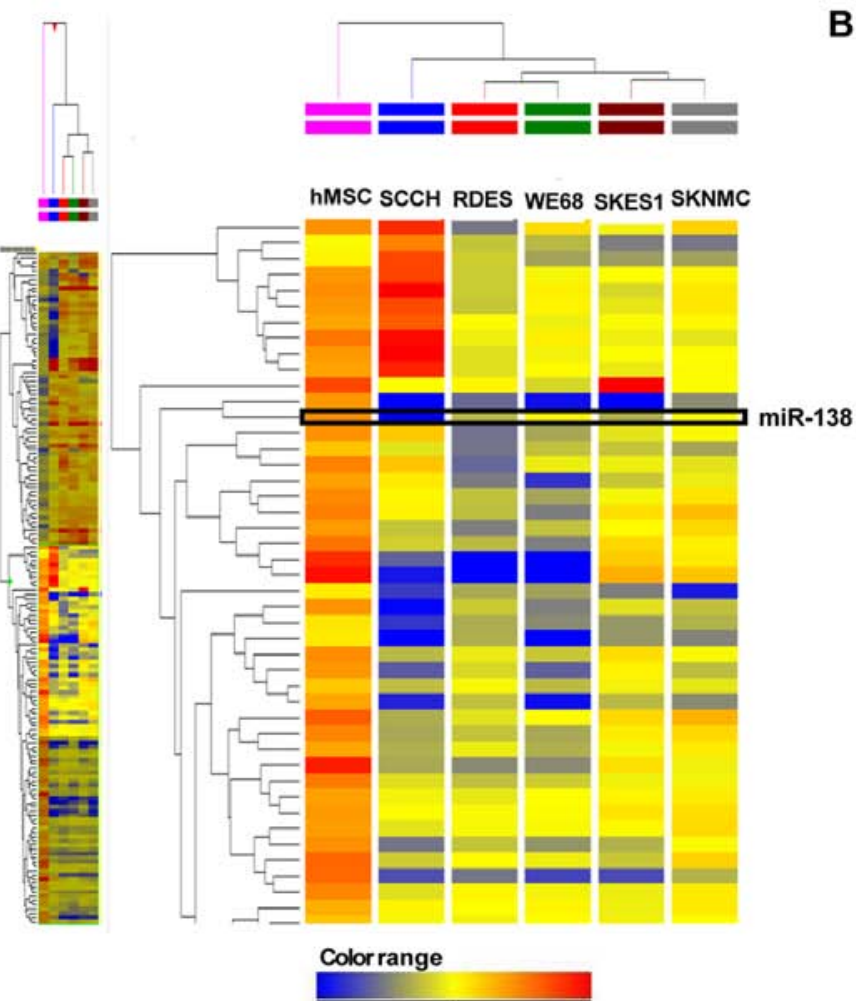

B

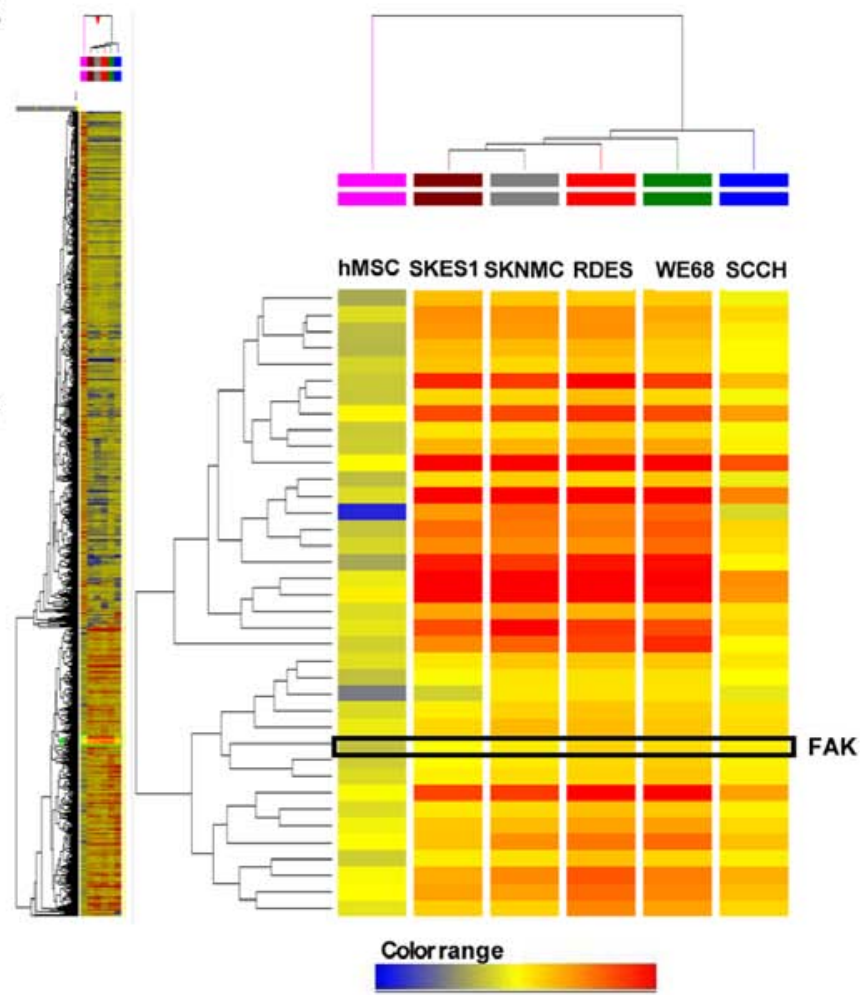

Figure 1. (A) Heat map of genome-wide miRNA profile in five ES cell lines and hMSCs. miR-138 is upregulated in all five ES cell lines. (B) Whole genome mRNA profile and extraction of FAK and related genes as the same pathways in ES cells and hMSCs.

A

Human PTK2 (NM_005607.4) ; 4437 bp

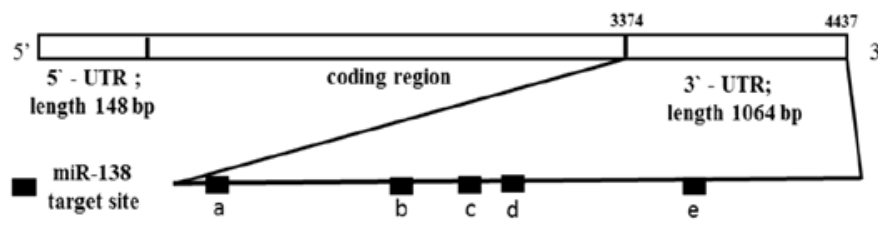

a $\begin{gathered}5^{\prime} \cdots \text { UCUUUUGGAGAUGUUCUCUAGC } \cdots 3 \\ \text { II I II II II } \\ \text { 3'- CCGGGACCACAACACUUCALCG - } 5\end{gathered}$ Position $30-52$ of $3^{\prime}$-UTR

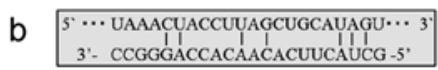
3'- CCGGGACCACAACACUUCAUCG -
Position $356-78$ of 3 ' UTR

C

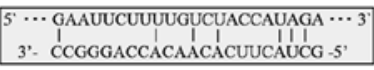

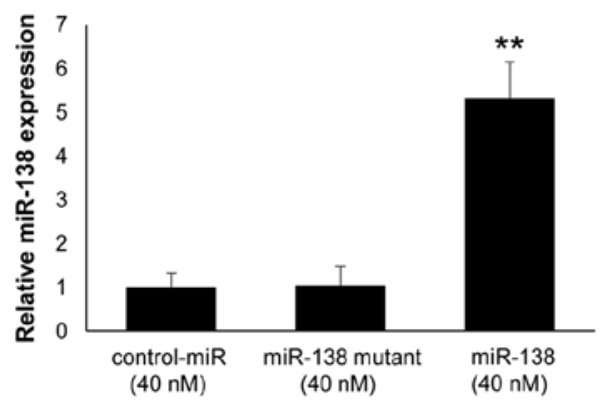

d

e

$5^{\circ} \cdots$ UAAUGUGCCAGUUUUUUGGUAGC $\cdots$
I ${ }^{\prime}$
3.- CCGGGACCACAACACUUCAUCG I I -5, Position $679-801$ of $3^{\circ}$-UTR

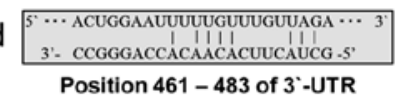

C

\section{Position $427-449$ of $3^{\circ}$-UTR}

$(40 \mathrm{nM})$

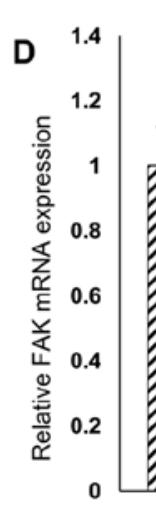

\section{B}

hsa-miR-138-1-3p sequence; WT; 3'- CCGGGACCACAACACUUCAUCG -5

hsa-mir-138-1-3p mutant sequence; Mut; 3'- CCGGGACCACAACUGAAGUAGG -5'
चControl-miR (40 nM)

- miR-138 mutant oligo (40 nM) BmiR-138 (40 nM)

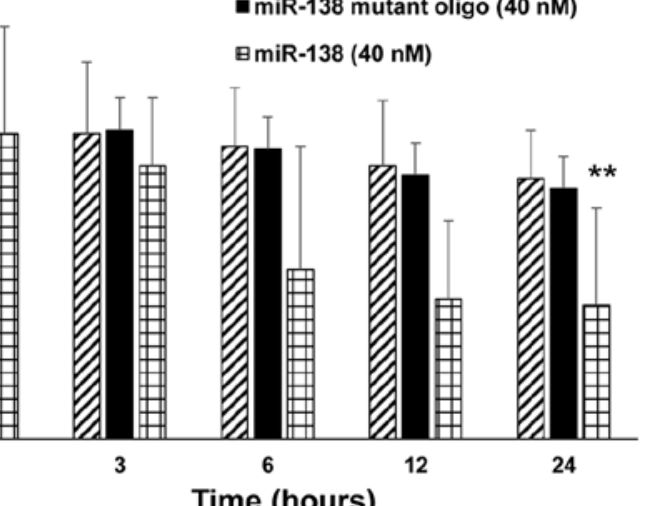

Figure 2. miR-138 inhibits FAK mRNA expression in SKES1 tumor cells. (A) Predicted binding sites of miR-138 (a-e) at the 3'-UTR site of FAK, as aligned by Target Scan and BLAST. (B) Scheme and sequence of the intact miR-138 (Wt) and its mutant (Mut). (C) The mRNA expression level of miR-138 in the negative control-miR, miR-138 and miR-138 mutant was measured by qRT-PCR. (D) The mRNA expression level of FAK in the negative control-miR, miR-138 and miR-138 mutant was measured by qRT-PCR. 
A

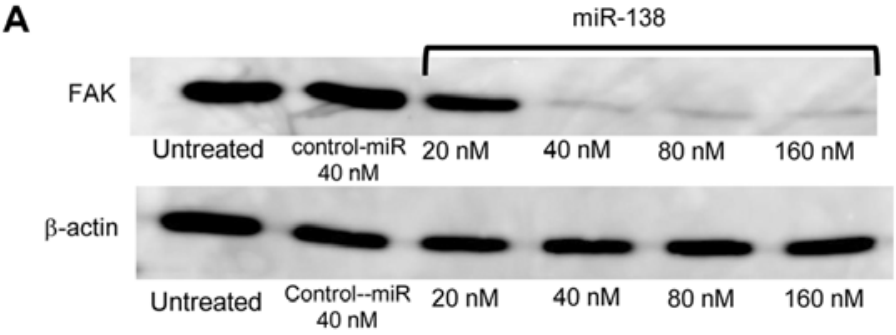

C
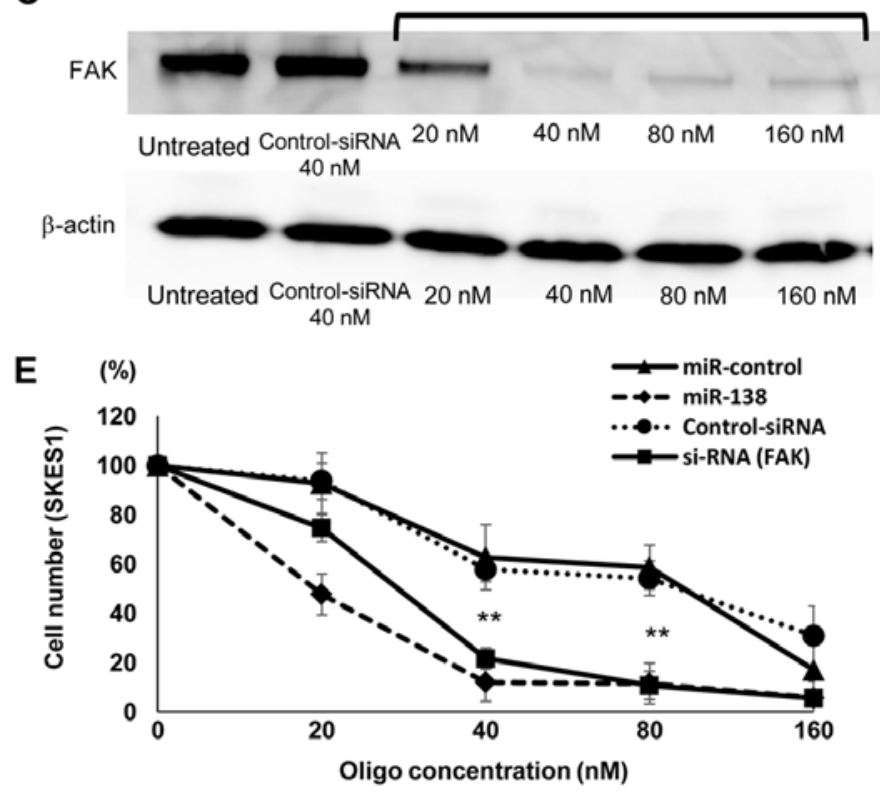

B

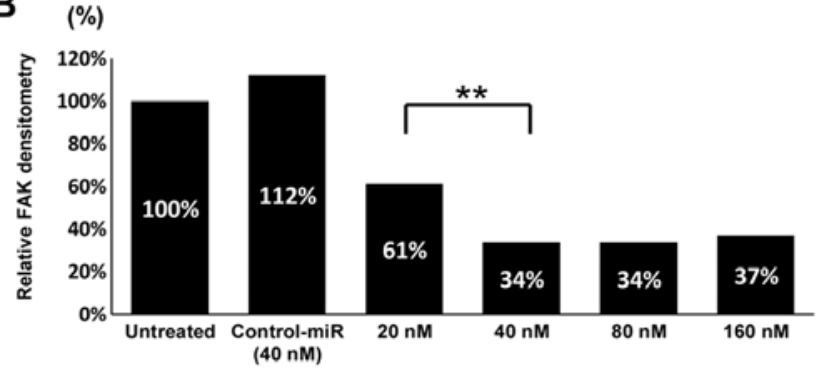

D
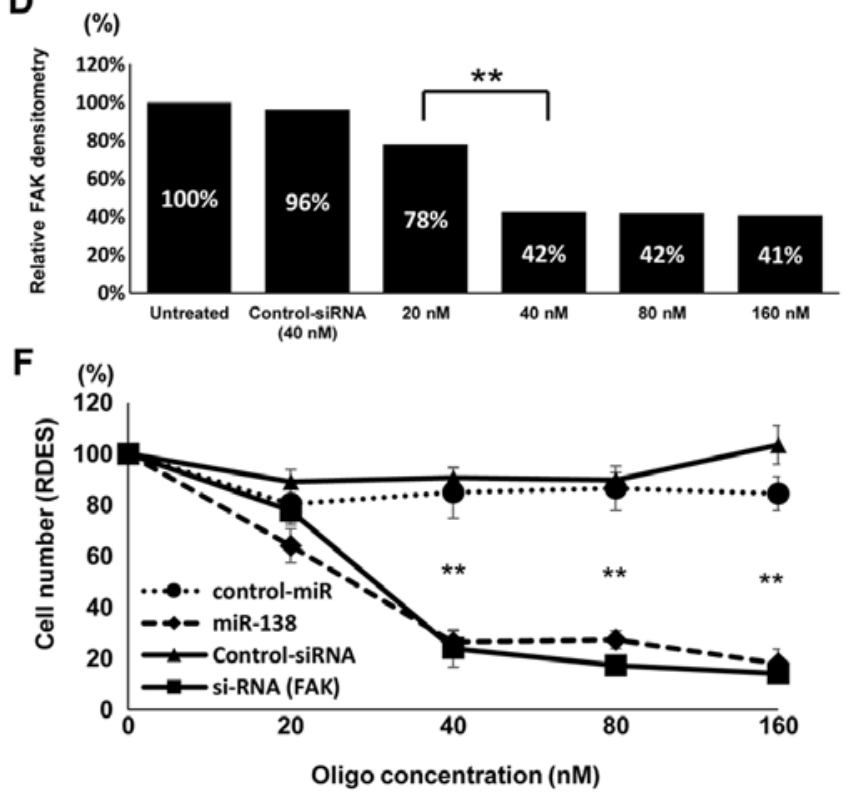

Figure 3. Silencing of FAK using miR-138 microRNA and FAK-siRNA in ES cells. (A) FAK protein is decreased in ES cells transfected with miR-138. (B) Densitometry quantification of FAK protein after transfection of miR-138. (C) Transfection of FAK-siRNA in ES cells reduces the expression of FAK protein. (D) The quantification of FAK protein after transfection of FAK-siRNA. ANOVA was carried out to statistically analyze the results. ${ }^{* * *} \mathrm{P}<0.01$. Cell proliferation assay to evaluate the antiproliferation effect of miR-138 and FAK-siRNA in SKES1 (E) and RDES (F) cells. Error bars represent mean \pm SD from three independent experiments. The two-tail Student's t-test was employed to statistically analyze the results: ${ }^{* *} \mathrm{P}<0.01$.

expression at the mRNA level following transfection with the miR-138-1-3p oligonucleotide (Fig. 2D).

Inhibition of FAK expression by miR-138 and FAK siRNA. To examine the correlation between miR-138 and FAK in ES cells, miR-138 was transfected into SKES1 cells. A western blot analysis showed that the expression level of FAK strongly decreased in miR-138 transfected cells compared to the untreated or negative control oligo-transfected cells (Fig. 3A). The FAK protein expression level in the miR-138 (40 nM) transfected cells was reduced to $34 \%$ of that in the control cells $(\mathrm{P}<0.01)$ (Fig. 3B). To further confirm the effects of FAK on the growth of ES cells, a transfection with siRNA-targeting FAK was carried out. Although the FAK protein expression level in the cells transfected with the negative control siRNA was not significantly affected, that in the cells transfected with FAK siRNA was significantly reduced, as determined by western blot analysis (Fig. 3C). Compared to the control cells (100\%), FAK siRNA (40 nM) transfected cells exhibited a significantly lower FAK expression level by $42 \%(\mathrm{P}<0.01)$ (Fig. 3D).

Suppression of ES cell growth by transfection of miR-138 and FAK siRNA. FAK is known to play important roles in the regulation of cell proliferation. As the transfection of miR-138 resulted in the reduction of FAK expression, we then examined the effects of miR-138 on the proliferation of ES cells. The SKES1 cell growth was inhibited by the transfection of miR-138 and FAK-siRNA compared to the negative control-miRNA and negative control-siRNA transfected cells, respectively, $48 \mathrm{~h}$ after the transfection, as determined by cell counts (Fig. 3E). RDES cells transfected with miR-138 and FAK siRNA (Fig. 3F) showed a significant inhibition of cell proliferation compared to the negative control-miR and siRNA-transfected cells. At the high concentration of 80 and $160 \mathrm{nM}$, the challenge of control miR and siRNA also exhibited growth inhibition in SKES1, but not in RDES cells. The results suggested that the susceptibility to toxicity of the oligonucleotides might differ among cell lines.

miR-138 attenuates the in vitro motility, migration and adhesion of ES cells through the targeting of FAK. We investigated the effects of miR-138 overexpression and target gene restoration on the motility and migration of SKES1 cells. In a Transwell migration assay, SKES1 cells stably transfected with miR-138 and a FAK-siRNA precursor presented impaired motility compared to those transfected with negative 
A
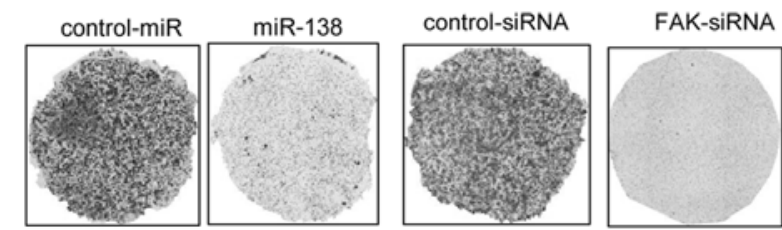

B
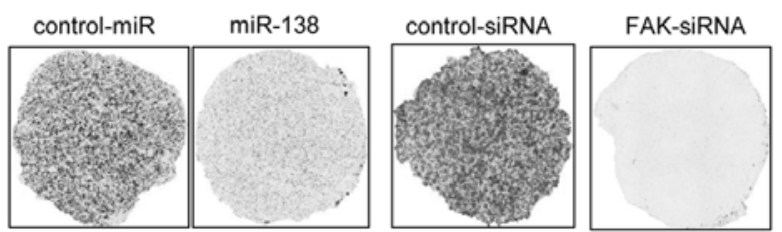

C

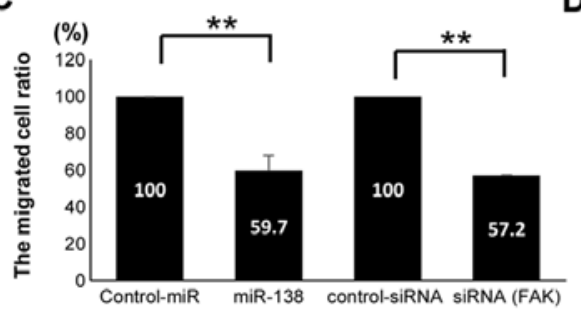

D
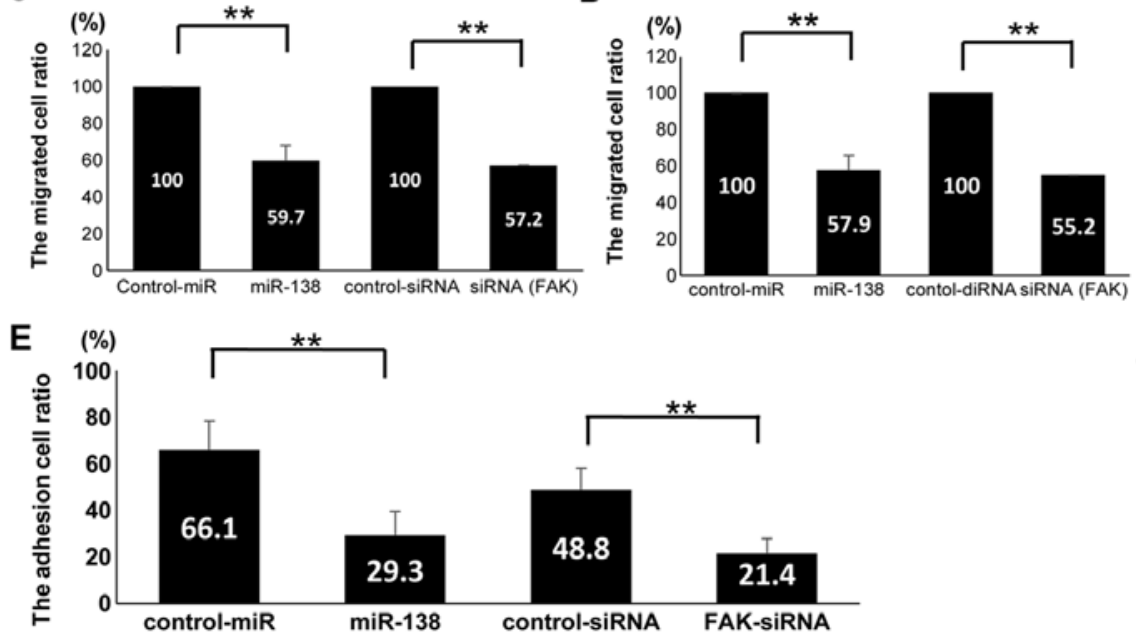

F

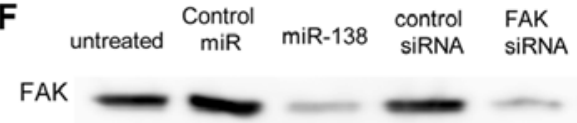

G

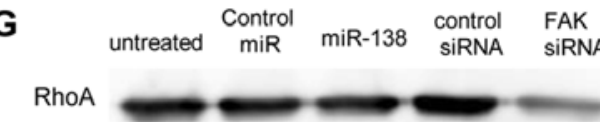

p-RhoA

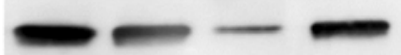

H
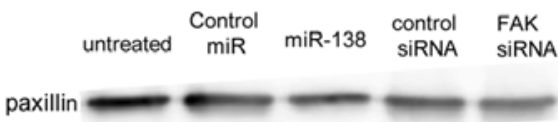

p-paxillin

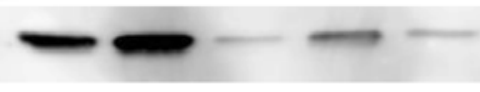

I untreated $\begin{gathered}\text { Control } \\ \text { miR }\end{gathered}$ miR-138 $\begin{array}{cc}\text { control } & \text { FAK } \\ \text { siRNA } & \text { siRNA }\end{array}$

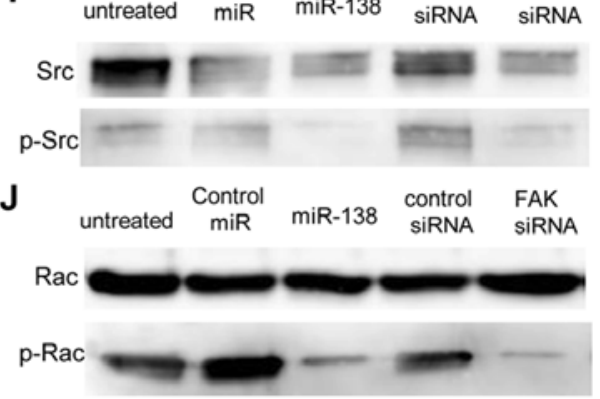

Figure 4. Transwell migration assay in SKES1 cells. ES cells stably transfected with miR-138 and a FAK-siRNA precursor showed impaired migration (A) and Transwell migration assay with Matrigel (B) compared to negative control-miR transfected cells. The quantification of the percentage of cells that migrated through the Transwell membrane (C) and a Matrigel Transwell assay (D) compared to negative control oligo-nucleotides in SKES cells. ANOVA test was carried out to statistically analyze the results. ${ }^{* *} \mathrm{P}<0.01$. The adhesion cell ratio following transfection with $40 \mathrm{nM}$ of miR-138 or FAK-siRNA, compared to negative control oligonucleotides cells in vitro (E). $\mathrm{P}<0.05,{ }^{* *} \mathrm{P}<0.01$. A western blot analysis of FAK (F), RhoA and p-RhoA (G), paxillin and p-paxillin (H), Src and p-Src (I), Rac and p-Rac (J) following the transfection of control-miR, miR-138, negative control siRNA or FAK-siRNA (40 nM) into SKES1 cells.

control-miR and control-siRNA (Fig. 4A). Transwell cells that went through the membrane without the Matrigel in miR-138 $(59.7 \pm 8.2 \%)$ and siRNAs $(57.2 \pm 7.1 \%)$ were significantly fewer than those of the negative control (Fig. 4C). In a Matrigel invasion assay, miR-138 overexpression and FAK-siRNA attenuated the invasiveness of SKES1 compared to the control-miRs (Fig. 4B). Transwell cells that went through the membrane with the Matrigel coated in miR-138 (57.9 $\pm 7.9 \%)$ and siRNAs $(55.2 \pm 6.4 \%)$ were significantly fewer than those of the negative control (Fig. 4D). To evaluate the cell adhesion ability, we counted the cells that did not float in vitro. Compared to the negative control miR $(66.1 \pm 12.2 \%)$ or the control siRNA group $(48.8 \pm 9.4 \%)$, the ratio of adhesion cells was lower in the miR138 $(29.3 \pm 10.3 \%)$ and the FAK-siRNA group $(24.1 \pm 6.3 \%)$, respectively (Fig. 4E). These results suggest that miR-138 and FAK-siRNA remarkably attenuated the in vitro migration and invasion of ES cells through the targeting of FAK. The expression level of FAK was markedly decreased in miR-138- (40 nM) and FAK-siRNA- (40 nM) transfected cells, than in the untreated or negative control oligo-transfected cells (Fig. 4F). The protein expression level of RhoA (Fig. 4G), paxillin (Fig. 4H), F-actin (data not shown),
Src (Fig. 4I) and Rac (Fig. 4J) did not significantly decrease in miR-138- (40 $\mathrm{nM})$ and FAK-siRNA- (40 nM) transfected cells, compared to the untreated or negative control oligotransfected cells. The phosphorylation level of RhoA, paxillin, Src and Rac significantly decreased in miR-138 (40 nM) and FAK-siRNA- (40 nM) transfected cells, compared to the untreated or negative control oligo-transfected cells.

Downregulation of FAK through miR-138- and siRNA-induced anoikis. In a fluorescence activated cell sorting (FACS) analysis of Annexin V-FITC/PI double staining, there was no significant difference between untreated, $40 \mathrm{nM}$ controlmiR, $40 \mathrm{nM}$ miR-138 and $40 \mathrm{nM}$ FAK-siRNA (Fig. 5A-D). We counted the number of floating cells following miR-138 treatment, to check the status of these excluded cells. Almost all of the cells that were floating following miR-138 treatment were sorted in the apoptosis fraction (Fig. 5E), rather than in the other adhesive cell groups. The effect of programmed cell death or apoptosis on SKES1 cells was not induced by miR-138 as anoikis in vitro. The cellular expression of PARP (Fig. 5F) and that of its cleaved product (Fig. 5G) was assayed by immunoblotting in the SKES1 cell lines and their transfec- 
A
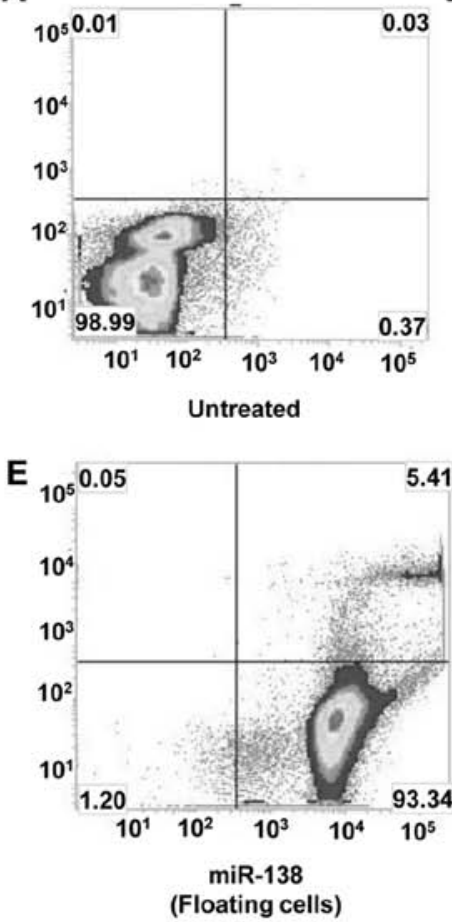

B

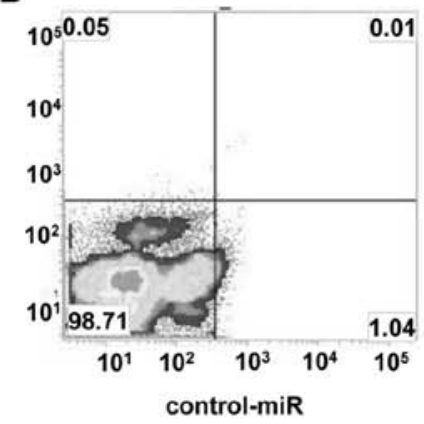

$F$

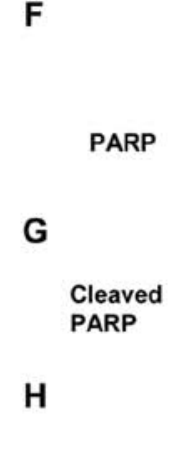

FAK
C

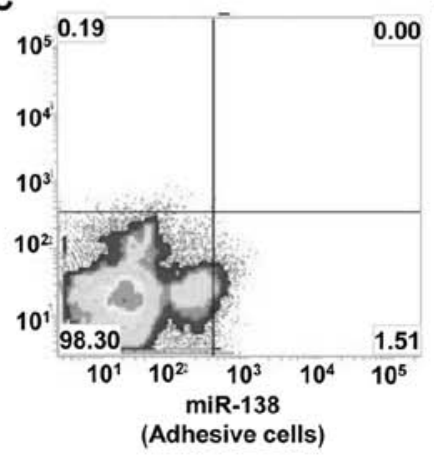

D

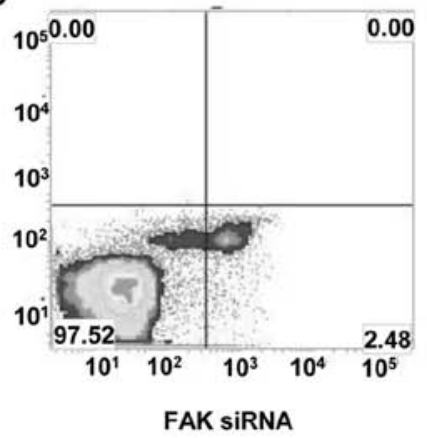

$\begin{array}{ccccc}\text { miR-138 } & \text { FAK- siRNA } & \text { miR-138 } & \text { FAK- siRNA } \\ \text { Untreated control-miR } & \text { (Adhesive cells) } & \text { (Adhesive cells) (Floating cells) } & \text { (Floating cells) }\end{array}$

Untreated control-miR (Adhesive cells) (Adhesive cells) (Floating cells) (Floating cells)
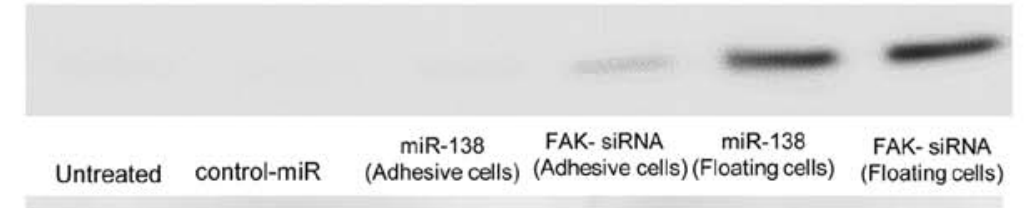

Unteated controlm

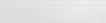

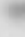

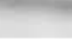

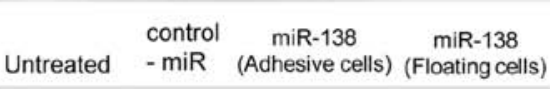

Untreated - miR (Adhesive cells) (Floating cells)

Figure 5. Effects of miR-138 and FAK-siRNA on induction of apoptosis in SKES1 cells. The cells were labelled with FITC Annexin V and PI (A-E). Each quadrants represent viable cells (lower left quadrant), early apoptotic cells (lower right), late or secondary necrotic cells (upper right), and primary necrotic cells (upper left), respectively. Western blot analysis shows the expression of PARP (F), its cleaved form (G) and FAK (H).

tions. The presence of the cleaved PARP product, a marker for caspase-mediated apoptosis, was not expressed in the miR-138 or siRNA against the FAK transfectants, nor in the untreated cells and negative control transfectants, in marked contrast to the cells that were floating following miR-138 and FAK-siRNA transfection (Fig. 5H).

miR-138 effects of cell cycle and apoptosis are dose-and time-dependent. To determine the cause of the repression of cell proliferation following the transfection of SKES1 cells with miR-138 at $20 \mathrm{nM}$ (Fig. 6A), $40 \mathrm{nM}$ (Fig. 6B), $80 \mathrm{nM}$ (C), $160 \mathrm{nM}$ (Fig. 6D), and with FAK-siRNA (Fig. 6E), we carried out a FACS analysis to measure the cell cycle distribution. In the 20-nM condition, the $\mathrm{S}$ and $\mathrm{G} 2 / \mathrm{M}$ phases were reduced, and the sub-G1 fraction was increased after $96 \mathrm{~h}$. Furthermore, we investigated the proportion of the sub-G1 fraction in PI in all groups, because the miR-138 transfectants were increased in the cells that were floating in the culture dish, and we only used adhesive cells in the cell count analysis. miR-138 might be a repressor of cell growth via the inhibition of cell adhesion, as the effect of miR-138 in SKES1 cells is the induction of anoikis in a dose- and time-dependent manner, resulting from the loss of the adhesive function in floating cells, and the repression of cell cycle progression in viable cells that were barely kept alive by adhesion. The proportion of cells in the sub-G0/G1 fraction was increased in a time- and miR-138 dose-dependent manner (Fig. 6G), whereas that of cells in the $\mathrm{S}$ phase (Fig. $6 \mathrm{H}$ ) and the G2/M (Fig. 6I) phase were decreased, also depending on the miR-138 dose and reaction time. The proportion of cells in the G0/G1 fraction showed no significant trend to be influenced by the ratio of cells in the sub G0/G1, S and G2/M fractions (Fig. 6G).

miR-138 inhibits tumor growth in a nude mouse xenograft model. Next, we investigated the efficacy of miR-138 against tumor growth in vivo. Stable transfection of miR-138 (Fig. 7C) and of a FAK-siRNA (Fig. 7D) precursor into SKES1 cells resulted in decreased growth of xenograft tumors from the tail vein of nude mice, compared to those stably transfected with untreated cells (Fig. 7A) or control-miR (Fig. 7B). In the experimental metastasis studies, mice treated with SKES1 cells stably transfected with miR-138 $\left(62.5 \pm 19.9 \mathrm{~mm}^{3}\right)$ and FAK-siRNA $\left(58.6 \pm 6.23 \mathrm{~mm}^{3}\right)$ displayed a statistically lower $(\mathrm{P}<0.01)$ lung metastatic volume than the untreated $\left(215.3 \pm 9.79 \mathrm{~mm}^{3}\right)$ and control-miR $\left(178.9 \pm 17 \mathrm{~mm}^{3}\right)$ group (Fig. 7E).

\section{Discussion}

miRNAs regulate gene expression post-transcriptionally by binding to the 3 '-UTR of their target mRNAs. Binding leads to the degradation of the target mRNAs and to reduced translation of the target proteins. miRNA activity also affects the expression of genes that are downstream of the direct targets, and can lead to changes in global protein expression profiles. Therefore, miRNAs potentially play a critical role in the progression of human cancer. Aberrant expression of miRNAs has been shown to play important roles in the 


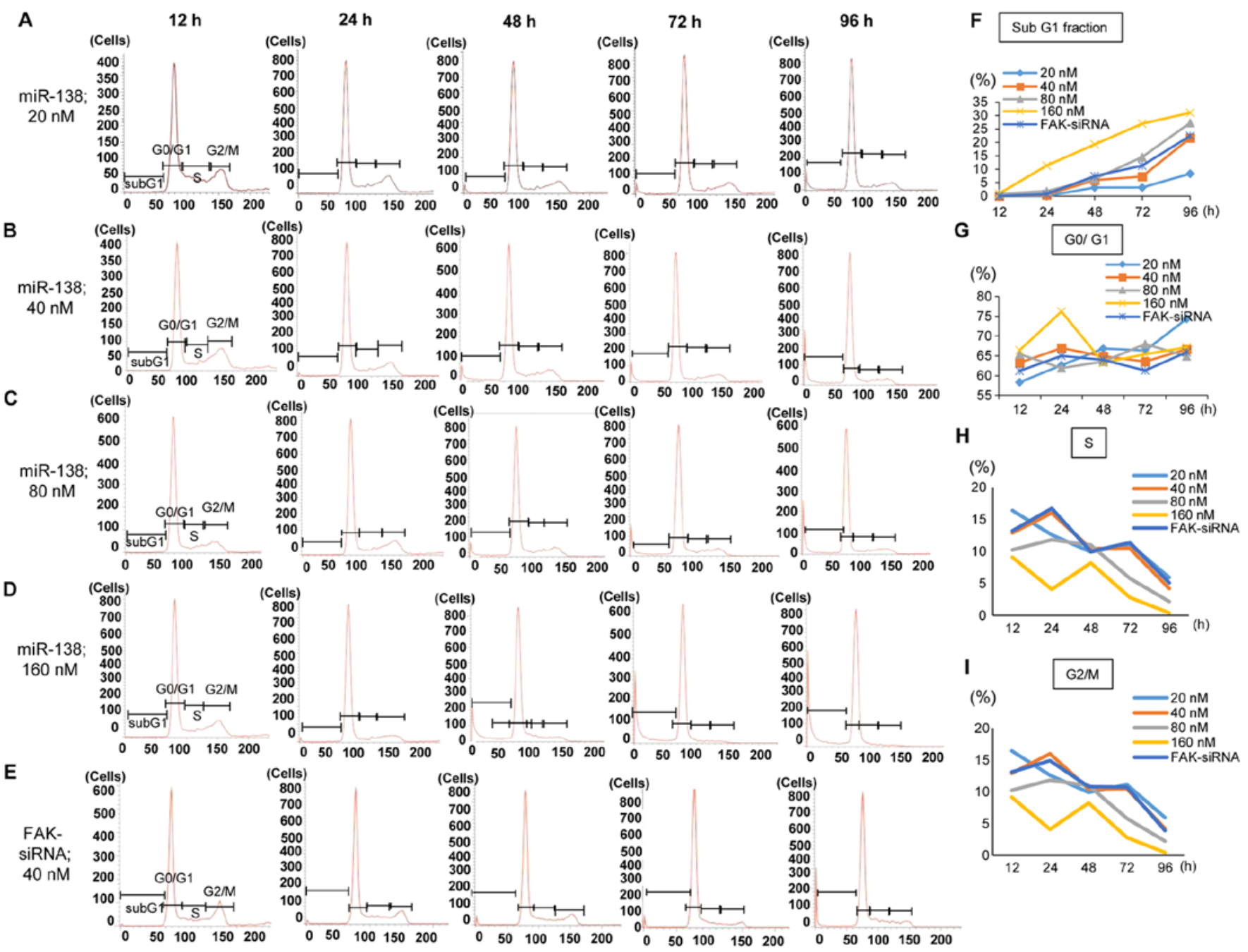

Figure 6. Effect of miR-138 on cell cycle in SKES1. Cells were treated and analyzed by flow cytometry after staining with PI. miR-138 concentration at $20 \mathrm{nM}$ (A), $40 \mathrm{nM}$ (B), $80 \mathrm{nM}$ (C), $160 \mathrm{nM}$ (D) and FAK-siRNA (E). The percentage of sub G1 fraction (F), G0/G1 (G), S phase (H) and G2/M phase (I).
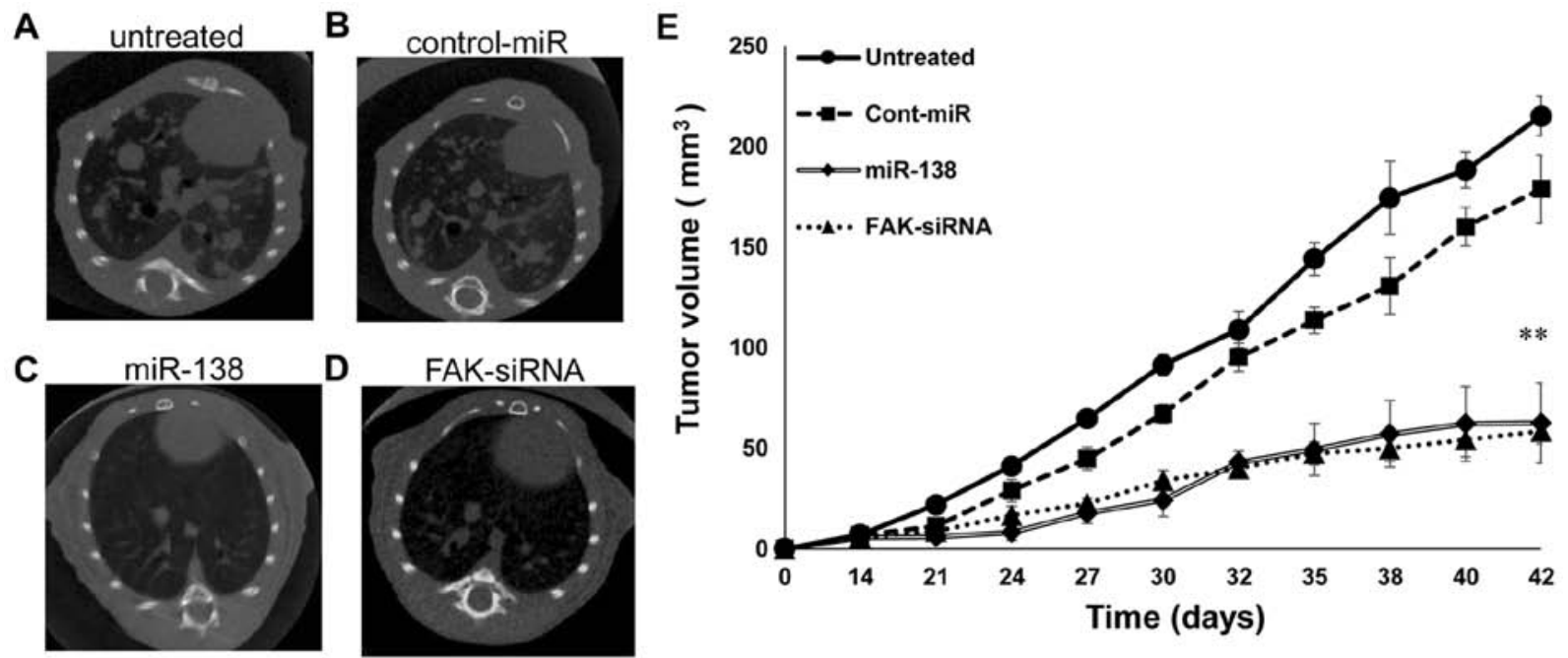

Figure 7. miR-138 suppresses in vivo tumor growth in SKES1. Untreated (A), negative control-miR (B), miR-138 (C) and FAK-siRNA transfected cells (D) were injected into the tail vein of nude mice to establish the tumor xenografts. Metastatic lesions $\left.{ }^{* * *} \mathrm{P}<0.01\right)$ in each group (E).

dysregulation of cell functions, including differentiation, proliferation, apoptosis, and metastasis $(20,21)$.
In the present study, our miRNA array results show that the expression of miR-138 was downregulated in all of the 
five ES cell lines. Several studies have shown that miR-138 is downregulated, and it is closely related to the abnormal potential of tumors $(11,21-23)$. The significant suppression of miR-138 expression in all of our five ES cell lines suggests that miR-138 might play a tumor suppressor role and might be related to some malignant potential in ES. However, neither the functional role nor the targets of miR-138 in ES have been previously defined. The results suggest that the expression of miR-138 was coordinately upregulated in ES cell lines, and prompted us to perform genome-wide mRNA profiling in these lines, using a cDNA array to detect the possible targets of miR-138 in ES cells.

The data from the cDNA array shows that FAK mRNA expression was increased in all of the five ES cell lines. FAK activity is regulated by cellular adhesion and downstream of growth factors and G-protein-linked receptor activation (24). It localizes to focal contact sites and receives input from both integrin and growth factor receptors. FAK is overexpressed in many malignant tumors, including lung cancer, and its expression level is linked to the poor prognosis of the corresponding cancers (25). Our data are consistent with these tumors in that upregulation of FAK may contribute to malignancy.

Although miR-138 might influence the expression of many genes, we focused on FAK as the target of miR-138 in ES cells. It has been reported that miR-138 targets several genes,

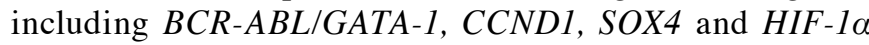
(10-12). Our cDNA array analysis demonstrated that FAK was the only miR-138-target gene whose expression was uniformly upregulated in all five ES cell lines, whereas the expression of other candidate genes varied among the ES cells. Using several algorithms, the analysis further suggested that FAK is the putative target of miR-138. Thus, we analyzed the possibility that miR-138 might contribute to anticancer activities by targeting FAK in ES cells.

Due to the reported importance of FAK in cancer (26), we further characterized its role in relation to miR-138. We examined the functions of miR-138 on the regulation of its predicted target genes and the changes in the biological characteristics of our ES cell lines. The forced elevation of miR-138 resulted in the reduction of the expression of the FAK protein, suggesting that miR-138 might function as a tumor suppressor gene in ES cells. Interestingly, FAK protein levels are downregulated by the overexpression of miR-138 are known as tumor suppressor gene. FAK, a protein tyrosine kinase involved in invasion and metastasis regulation, is a direct target of miR-138 (27), and FAK has the ability to promote cell cycle progression (14-16). Aberrant expression of FAK will lead to abnormal cellular proliferation. Our results suggest that the same mechanism of regulation of FAK expression by miR-138 might exist in ES cell lines.

FAK plays an important role in the regulation of cellular invasion, migration and metastasis (28). Downregulation of FAK expression by miR-138 inhibited cell attachment, invasion and migration compared to transfection negative control-miR in SKES cells. Our motility analyses showed that miR-138 inhibited the movement of ES cells by repressing motility. These observations are consistent with previous reports demonstrating that FAK is necessary for its motility (29). We can assume that upregulation of miR-138 regulates the movement of ES cells via miR-138-mediated FAK.
The percentage of apoptosis was increased following transfection of $40 \mathrm{nM}$ of miR-138, the tumor growth of which was repressed in vitro. We found that cells are divided into adherent cells and floating cells in petri dishes that were transfected with miR-138. To verify that the repression of FAK via miR-138 and FAK-siRNA could induce apoptosis, we collected the floating cells following transfection of miR/siRNA, and analyzed them to compare with the adhered cells in the culture dishes. We showed that knocking down through the upregulation of miR-138 and the transfection of FAK-siRNA induced anoikis in floating cells. However, apoptosis was not induced in the adhered cells that were transfected with miR-138/ FAK-siRNA, based on the data on Annexin V and the expression of the cleaved PARP protein.

Duration of $48 \mathrm{~h}$ and a concentration of $40 \mathrm{nM}$ represent the optimal miR-138 transfection conditions for FAK expression, in which the number of tumor cells is reduced, but it is monistic. To examine the function of miR-138 and the biology of FAK in SKES1 in more detail, we tried to change the reaction time and concentration of miR-138. In miR-138-transfected SKES1 cells, the cell cycle progression was suppressed in adhesive cells and apoptosis was simultaneously induced in floating cells. miR-138 exerts its antitumorigenic action in two ways: one is the repression of the adhesive function, which leads to anoikis, and the other is the repression of the cell cycle in viable cells that are barely holding on the ground. Furthermore, these two methods depend on the duration time and the concentration.

The overexpression of miR-138 in SKES1 cells resulted in the inhibition of metastatic lung tumor growth in mouse xenograft models. This study shows an important tumor suppressor role in metastasis through the transfection of miR-138 in SKES1 cells.

In conclusion, the present study suggests for the first time a correlation between miR-138 and FAK in ES cells. Our results provide evidence that the expression level of miR-138 in ES cells was significantly reduced and inversely correlated with the FAK expression level, and that miR-138 plays important roles in ES cell proliferation, invasion, and migration by targeting FAK both in vitro and in vivo. Our data suggest that FAK is one of the crucial factors that enhance tumor proliferation, invasion, and migration in ES, as well as in other malignant tumors. Recent studies have shown that FAK plays critical roles in host cells in the tumor microenvironment and in the tumor cells that contribute to metastasis $(30,31)$. FAK plays pleiotropic roles in cancer, and this research has to progress to the next step, i.e., until the phenomenon is also confirmed in human tumor tissue samples. This study is the first report showing that miR-138 inhibits the proto-oncogenic FAKs, providing deeper insight into the biology of ES tumorigenesis. Our results might therefore prove useful to clinical work in the future.

\section{Acknowledgements}

The authors thank Dr Katsuhiro Hanada and Dr Takashi Kobayashi, for helpful discussion for this study. This study was supported in part by National Cancer Center Research and Development Fund (26-A-4), the Grants-in-Aid for Scientific Research (no. 24592250) and 15K10451 from Japan Society 
for the Promotion of Science, and the Grants-in-Aid for Scientific Research from Japan Agency for Medical Research and Development (15Ack0106087h0002)..

\section{References}

1. Bleyer A, Barr R, Hayes-Lattin B, Thomas D, Ellis C and Anderson B; Biology and Clinical Trials Subgroups of the US National Cancer Institute Progress Review Group in Adolescent and Young Adult Oncology: The distinctive biology of cancer in adolescents and young adults. Nat Rev Cancer 8: 288-298, 2008.

2. Taylor R, Knowles HJ and Athanasou NA: Ewing sarcoma cells express RANKL and support osteoclastogenesis. J Pathol 225: 195-202, 2011.

3. Paulussen M, Craft AW, Lewis I, Hackshaw A, Douglas C Dunst J, Schuck A, Winkelmann W, Köhler G, Poremba C, et al; European Intergroup Cooperative Ewing's Sarcoma Study-92: Results of the EICESS-92 Study: Two randomized trials of Ewing's sarcoma treatment - cyclophosphamide compared with ifosfamide in standard-risk patients and assessment of benefit of etoposide added to standard treatment in high-risk patients. J Clin Oncol 26: 4385-4393, 2008.

4. Ladenstein R, Pötschger U,Le Deley MC, Whelan J, Paulussen M, Oberlin O, van den Berg H, Dirksen U, Hjorth L, Michon J, et al: Primary disseminated multifocal Ewing sarcoma: Results of the Euro-EWING 99 trial. J Clin Oncol 28: 3284-3291, 2010.

5. Meltzer PS: Cancer genomics: Small RNAs with big impacts. Nature 435: 745-746, 2005.

6. Esquela-Kerscher A and Slack FJ: Oncomirs - microRNAs with a role in cancer. Nat Rev Cancer 6: 259-269, 2006.

7. Lu J, Getz G, Miska EA, Alvarez-Saavedra E, Lamb J, Peck D, Sweet-Cordero A, Ebert BL, Mak RH, Ferrando AA, et al: MicroRNA expression profiles classify human cancers. Nature 435: 834-838, 2005.

8. Yeh YM, Chuang CM, Chao KC and Wang LH: MicroRNA-138 suppresses ovarian cancer cell invasion and metastasis by targeting SOX4 and HIF-1 $\alpha$. Int J Cancer 133: 867-878, 2013

9. Zhang H, Zhang H, Zhao M, Lv Z, Zhang X, Qin X, Wang H, Wang S, Su J, Lv X, et al: MiR-138 inhibits tumor growth through repression of EZH2 in non-small cell lung cancer. Cell Physiol Biochem 31: 56-65, 2013.

10. Xu C, Fu H, Gao L, Wang L, Wang W, Li J, Li Y, Dou L, Gao X, Luo X, et al: BCR-ABL/GATA1/miR-138 mini circuitry contributes to the leukemogenesis of chronic myeloid leukemia. Oncogene 33: 44-54, 2014.

11. Liu X, Lv XB, Wang XP, Sang Y, Xu S, Hu K, Wu M, Liang Y, Liu P, Tang J, et al: MiR-138 suppressed nasopharyngeal carcinoma growth and tumorigenesis by targeting the CCND1 oncogene. Cell Cycle 11: 2495-2506, 2012.

12. Kohlhoff J and Barnett B: Parenting self-efficacy: Links with maternal depression, infant behaviour and adult attachment. Early Hum Dev 89: 249-256, 2013.

13. Schlaepfer DD and Mitra SK: Multiple connections link FAK to cell motility and invasion. Curr Opin Genet Dev 14: 92-101, 2004.

14. Siesser PM and Hanks SK: The signaling and biological implications of FAK overexpression in cancer. Clin Cancer Res 12: 3233-3237, 2006.

15. McLean GW, Carragher NO, Avizienyte E, Evans J, Brunton VG and Frame MC: The role of focal-adhesion kinase in cancer - a new therapeutic opportunity. Nat Rev Cancer 5: 505-515, 2005.
16. Lahlou H, Sanguin-Gendreau V, Zuo D, Cardiff RD, McLean GW, Frame MC and Muller WJ: Mammary epithelialspecific disruption of the focal adhesion kinase blocks mammary tumor progression. Proc Natl Acad Sci USA 104: 20302-20307, 2007.

17. Kong X, Li G, Yuan Y, He Y, Wu X, Zhang W, Wu Z, Chen T, Wu W, Lobie PE, et al: MicroRNA-7 inhibits epithelial-tomesenchymal transition and metastasis of breast cancer cells via targeting FAK expression. PLoS One 7: e41523, 2012.

18. Song J, Kim D and Jin EJ: MicroRNA-488 suppresses cell migration through modulation of the focal adhesion activity during chondrogenic differentiation of chick limb mesenchymal cells. Cell Biol Int 35: 179-185, 2011.

19. Luedde T: MicroRNA-151 and its hosting gene FAK (focal adhesion kinase) regulate tumor cell migration and spreading of hepatocellular carcinoma. Hepatology 52: 1164-1166, 2010.

20. Lujambio A and Esteller M: How epigenetics can explain human metastasis: A new role for microRNAs. Cell Cycle 8: 377-382, 2009.

21. Medina PP and Slack FJ: microRNAs and cancer: An overview. Cell Cycle 7: 2485-2492, 2008.

22. Wang W, Zhao LJ, Tan YX, Ren H and Qi ZT: MiR-138 induces cell cycle arrest by targeting cyclin D3 in hepatocellular carcinoma. Carcinogenesis 33: 1113-1120, 2012.

23. Lee YC, Tzeng WF, Chiou TJ and Chu ST: MicroRNA-138 suppresses neutrophil gelatinase-associated lipocalin expression and inhibits tumorigenicity. PLoS One 7: e52979, 2012.

24. Schlaepfer DD, Hauck CR and Sieg DJ: Signaling through focal adhesion kinase. Prog Biophys Mol Biol 71: 435-478, 1999.

25. Carelli S, Zadra G, Vaira V, Falleni M, Bottiglieri L, Nosotti M, Di Giulio AM, Gorio A and Bosari S: Up-regulation of focal adhesion kinase in non-small cell lung cancer. Lung Cancer 53: 263-271, 2006

26. Siejka A, Barabutis N and Schally AV: GHRH antagonist inhibits focal adhesion kinase (FAK) and decreases expression of vascular endothelial growth factor (VEGF) in human lung cancer cells in vitro. Peptides 37: 63-68, 2012.

27. Golubovskaya VM, Sumbler B, Ho B, Yemma M and Cance WG: MiR-138 and MiR-135 directly target focal adhesion kinase, inhibit cell invasion, and increase sensitivity to chemotherapy in cancer cells. Anticancer Agents Med Chem 14: 18-28, 2014.

28. Nowicki TS, Zhao H, Darzynkiewicz Z, Moscatello A, Shin E, Schantz S, Tiwari RK and Geliebter J: Downregulation of uPAR inhibits migration, invasion, proliferation, FAK/PI3K/Akt signaling and induces senescence in papillary thyroid carcinoma cells. Cell Cycle 10: 100-107, 2011.

29. Li LH, Zheng MH, Luo Q, Ye Q, Feng B, Lu AG, Wang ML, Chen XH, Su LP and Liu BY: P21-activated protein kinase 1 induces colorectal cancer metastasis involving ERK activation and phosphorylation of FAK at Ser-910. Int J Oncol 37: 951-962, 2010.

30. Jia YL, Shi L, Zhou JN, Fu CJ, Chen L, Yuan HF, Wang YF, Yan XL, Xu YC, Zeng Q, et al: Epimorphin promotes human hepatocellular carcinoma invasion and metastasis through activation of focal adhesion kinase/extracellular signal-regulated kinase/matrix metalloproteinase-9 axis. Hepatology 54: 1808-1818, 2011.

31. Fan H, Zhao X, Sun S, Luo M and Guan JL: Function of focal adhesion kinase scaffolding to mediate endophilin A2 phosphorylation promotes epithelial-mesenchymal transition and mammary cancer stem cell activities in vivo. J Biol Chem 288: 3322-3333, 2013 